\section{Surface tension measurements with a smartphone}

\section{Nicolas-Alexandre Goy, Zakari Denis, Maxime Lavaud, Adrian Grolleau, Nicolas Dufour, Antoine Deblais, and Ulysse Delabre, University of Bordeaux, France}

martphones are increasingly used in higher education and $\checkmark$ at university in mechanics, ${ }^{1-3}$ acoustics, ${ }^{4}$ and even thermodynamics ${ }^{5}$ as they offer a unique way to do simple science experiments. In this article, we show how smartphones can be used in fluid mechanics to measure surface tension of various liquids, which could help students understand the concept of surface tension through simple experiments.

\section{Background}

Interfacial tension is the energy per unit area required for a material to create an interface with a surrounding material. This surface tension exists for interfaces between solids, liquids, and gas. In the case of a liquid surrounded by air, interfacial tension is often called surface tension, which plays an essential role in many natural phenomena such as pulmonary breathing, use of detergents, and insect-walking on water. ${ }^{6-8}$ Usually surface tension is measured with quite expensive equipment (Wilhelmy plate, ring method) and long calibration procedures in order to get very accurate values, which perhaps prevents a simple understanding of the concept of surface tension. The method described here is based on the standard pendant drop method, ${ }^{9}$ where a drop of liquid is suspended from a tube as shown in Fig. 1(a). The shape of the drop is then governed by the balance of surface tension forces along the tube and the weight of the drop. We show here that without a specific image setup, smartphones are accurate enough to get reasonable values of surface tension for various liquids such as water, oil, and water with detergent, and can be done easily by students on their smartphones.

When a drop is hung at the tip of a tube, the shape of the drop is governed by the local Laplace equation, where the weight of the drop and surface tension forces are balanced. The complete resolution of this equation requires computa-

tional ${ }^{9,10}$ analysis, which is the basis of the commercial measurement of surface tension.

However another way to estimate surface tension $\gamma$ can be given by the following simple equation ${ }^{10}$ :

$$
\gamma=\frac{\Delta \rho g D_{E}^{2}}{H},
$$

where $\Delta \rho$ is the difference in density between the liquid and air, $g$ is the gravitational constant $\left(g=9.81 \mathrm{~m} / \mathrm{s}^{2}\right), D_{\mathrm{E}}$ is defined in Fig. 1(a) and is the maximum diameter of the pendant drop, and

$$
\frac{1}{H}=f\left(\frac{D_{S}}{D_{E}}\right)
$$

is a dimensionless function of the ratio between $D_{\mathrm{S}}$ and $D_{\mathrm{E}}$, which accounts for the specific shape of the drop due to gravity. $D_{\mathrm{S}}$ is defined as the diameter of the drop at a distance $D_{\mathrm{E}}$ from the bottom of the drop. For instance, if the drop was a perfect sphere, $D_{\mathrm{S}}$ would be zero. Due to gravity, the droplet is then elongated and $D_{\mathrm{S}}$ becomes strictly positive. This $1 / H$ function can then be understood as a form factor and has been calculated numerically, ${ }^{9}$ but for our purpose we use the values tabulated in classic books ${ }^{10}$ and represented in Fig. $1(\mathrm{c})$. However, it is also interesting to note that within a good approximation, this $1 / H$ dimensionless function can be approximated by a simple analytical formula,

$$
\frac{1}{H}=a\left(\frac{D_{S}}{D_{E}}\right)^{b},
$$

with $a \approx 0.345$ and $b \approx-2.5$.

Thus, following Eq. (1), surface tension can be obtained by measuring diameters with a ruler directly on the screen of the smartphone using the optical and numerical zooms of the smartphone.

\title{
Experiment
}

Droplets of various liquids (water, olive oil, water with surfactants, ethanol) were suspended at the tip of a Pasteur pipette (diameter tip $d_{\text {Tube }}=1.4 \mathrm{~mm}$ ) by dipping it and pulling it out of a liquid reservoir. The

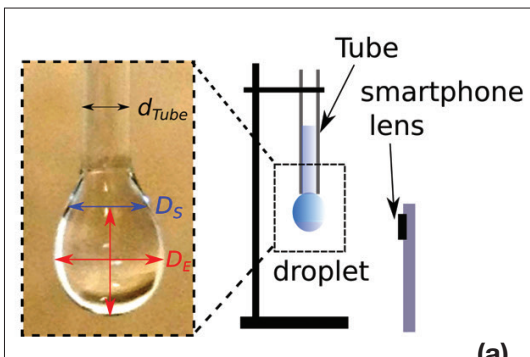

(a)

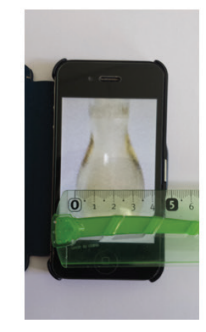

(b)



Fig. 1. (a) Water droplet suspended at the tip of a Pasteur pipette. (b) Illustration of the measurement of the diameters directly on the screen of the smartphone with a simple ruler. (c) $1 / H$ as a function of the ratio $D_{\mathrm{S}} / D_{\mathrm{E}} \cdot{ }^{10}$ Note the logarithmic scale on the axes. experiment has also been tested with standard straws such as the tube of a pen $\left(d_{\text {Tube }} \approx 2.9 \mathrm{~mm}\right)$ or McDonald's straw $\left(d_{\text {Tube }} \approx 5.9 \mathrm{~mm}\right)$ instead of Pasteur pipette to test the generality of our approach. The smartphone used in the experiment is an iPhone $4 \mathrm{~s}$ (camera sensor 8 MPixels, display $640 \times 960$ pixels, 3.5-in screen) and the standard camera function of the smartphone 

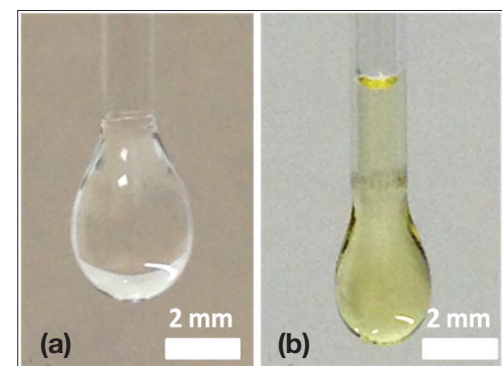
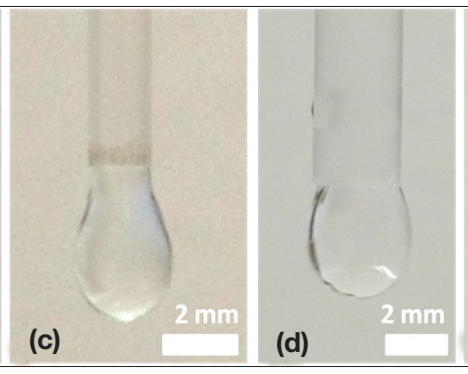

Fig. 2. (a) Water droplet, (b) oil droplet, (c) ethanol droplet hung at the tip of a Pasteur pipette. (d) Water droplet hung at the tip of a pen tube. (e) Water droplet hung at the tip of a McDonald's straw.

Table I. Surface tension values obtained with the smartphone pendant drop method.

\begin{tabular}{|c|c|c|c|c|}
\hline & $\begin{array}{c}\text { Typical } \\
\text { range of } \\
D_{\mathrm{S}} / D_{\mathrm{E}}\end{array}$ & $\begin{array}{c}\text { Density } \\
\text { differ- } \\
\text { ence } \\
\Delta \rho= \\
\rho_{\mathrm{L}}-\rho_{\text {air }} \\
\left(\mathbf{k g} / \mathbf{m}^{3}\right)\end{array}$ & \begin{tabular}{|c|} 
Surface \\
tension \\
$(\mathrm{mN} / \mathrm{m})$ \\
$($ Smartphone \\
measure- \\
ment at \\
$\left.20^{\circ} \mathrm{C}\right)$ \\
\end{tabular} & \begin{tabular}{|l|} 
Surface \\
tension \\
tabulated \\
value \\
$(\mathrm{mN} / \mathrm{m})$ \\
at $20^{\circ} \mathrm{C}$
\end{tabular} \\
\hline $\begin{array}{l}\text { Water (Pasteur } \\
\text { pipette) }\end{array}$ & $0.68-0.78$ & 999 & $\begin{array}{l}78 \pm 15 \\
\text { (6 drops) }\end{array}$ & 72.8 \\
\hline $\begin{array}{l}\text { Olive oil } \\
\text { Pasteur pipette) }\end{array}$ & 0.75 & 930 & $\begin{array}{c}33 \pm 3 \\
\text { (2 drops) }\end{array}$ & 32 \\
\hline $\begin{array}{l}\text { Ethanol Pasteur } \\
\text { pipette) }\end{array}$ & 0.8 & 780 & $\begin{array}{c}22 \pm 2 \\
\text { (2 drops) }\end{array}$ & 23 \\
\hline $\begin{array}{l}\text { Water (pen } \\
\text { tube) }\end{array}$ & 0.82 & 999 & 60 (2 drops) & 72.8 \\
\hline $\begin{array}{l}\text { Water } \\
\text { McDonald's } \\
\text { straw) }\end{array}$ & $\sim 1$ & 999 & 67 & 72.8 \\
\hline
\end{tabular}

is used. Taking advantage of the optical lens and the digital zoom (x5) of the smartphone, it is possible to measure $D_{\mathrm{E}}$, $D_{S}$, and $d_{\text {Tube }}$ directly on the screen of the smartphone with a standard ruler. For example, a droplet with a real diameter $D_{\mathrm{E}}=3.3 \mathrm{~mm}$ [Fig. 1(a)] can be magnified using the optics and the digital zoom of the smartphone into a $2.8-\mathrm{cm}$ diameter, which can easily be measured. For more accurate measurements, it is also possible to export pictures and analyze them with the free image analysis software ImageJ. ${ }^{11}$

\section{Results}

Figure 2 shows typical pictures of various droplets at room temperature $\left(20^{\circ} \mathrm{C}\right)$ taken with the smartphone. Using Eq. (1) we get surface tension values summarized in Table I. These results show that relatively good surface tension values for various liquids are obtained with this smartphone method. Even if the values obtained for surface tension for water are scattered, the values are always larger than the surface tension of olive oil and ethanol droplets, which is consistent with literature data. Indeed, a rough estimate of surface tension is given by ${ }^{12}$

$$
\gamma \sim \frac{E}{a^{2}}
$$

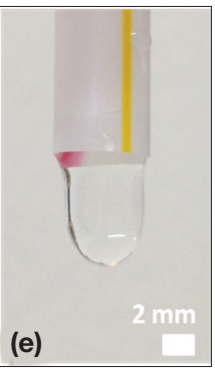

where $E$ is the typical energy between two molecules and $a$ the typical size of the molecules. This explains that the surface tension of water due to hydrogen bonds is much higher than that of olive oil or ethanol.

For other measurements with standard straws, the experimental surface tension gives reasonable values but they are less accurate as explained in Ref. 13 because the diameter of the tube is quite large, especially compared to capillary length

$$
L_{\mathrm{c}}=\sqrt{\frac{\gamma}{\Delta \rho g}},
$$

which induces $D_{\mathrm{S}} / D_{\mathrm{E}}$ ratios close to 1 [see for example Fig. $2(\mathrm{e})]$.

\section{Conclusion}

We present here a simple method to measure the surface tension of various liquids with a smartphone. The simplicity of our method especially compared to force measurement methods makes this approach interesting for educational purposes. It could enable a simple characterization of surface tension.

\section{References}

1. P. Vogt and J. Kuhn, "Analyzing simple pendulum phenomena with a smartphone acceleration sensor," Phys. Teach. 50, 439 (Oct. 2012).

2. P. Vogt and J. Kuhn, "Analyzing free fall with a smartphone acceleration sensor," Phys. Teach. 50, 182 (March 2012).

3. L. Madani, S. Ledenmat, A. Bsiesy, and J. Chevrier, "Teaching classical mechanics using smartphones," Phys. Teach. 51, 376 (Sept. 2013).

4. M. Hirth, J. Kuhn, and A. Müller, "Measurement of sound velocity made easy using harmonic resonant frequencies with everyday mobile technology," Phys. Teach. 53, 120 (Feb. 2015).

5. R. Elizabeth Vieyra, C. Vieyra, and S. Macchia, "Kitchen physics: Lessons in fluid pressure and error analysis," Phys. Teach. 55, 87 (Feb. 2017).

6. D. L. Hu and J. W. M. Bush, "Meniscus climbing insects," Nat. 437, 733-736 (2005).

7. Mohamed Boutinguiza Larosi, "Floating together on the top," Phys. Teach. 53, 93 (Feb. 2015).

8. Renate J. Ondris-Crawford and Lisa Hilliard, "It's all on the surface," Phys. Teach. 35, 100 (Feb. 1997).

9. C. E. Stauffer, "The measurement of surface tension by the pendant drop technique," J. Phys. Chem. 69, 1933 (1965).

10. A.W. Adamson, Physical Chemistry of Surfaces, 2nd ed. (Interscience Publishers, 1967).

11. ImageJ software, https://imagej.nih.gov/ij/ .

12. M. V. Berry, "The molecular mechanism of surface tension," Phys. Educ. 6 (2), 79-84 (1971).

13. J. D. Berry, M. J. Neeson, R. R. Dagastine, D. Y. Chan, and R. F. Tabor, "Measurement of surface and interfacial tension using pendant drop tensiometry," J. Colloid. Interface Sci. 454, 226 (2015). 